\title{
Bargmann-Radon transform for axially monogenic functions
}

\author{
Alí Guzmán Adán, Ren Hu*, Tim Raeymaekers, Franciscus Sommen
}

\begin{abstract}
In this paper we study the Bargmann-Radon transform and a class of monogenic functions called axially monogenic functions. First we compute the explicit formula of the Bargmann-Radon transform for axially monogenic functions, by making use of the Funk-Hecke Theorem. Then we present the explicit form of the general Cauchy-Kowalewski extension for radial function. Finally by making use of the results we obtained, we give an application of the Bargmann-Radon transform for Cauchy-Kowalewski extension.
\end{abstract}

Keywords: Clifford Analysis, Bargmann-Radon transform, Axially monogenic functions, Cauchy-Kowalewski extension

Mathematics Subject Classification (2010): 44A12; 30G35

\section{Introduction}

Johann Radon first introduced the Radon transform as an integral transform over a line, see $[11,18]$. The Radon transform is widely used in pure mathematics e.g. partial differential equations as well as applied mathematics e.g. tomography and X-ray transforms, e.g. [9, 24]. Therefore, it is an essential tool in technologies in medicine and engineering. The transform has also been generalized into higher-dimensional Euclidean spaces and provided some new insights in domains such as geometry and functional analysis, see e.g. [5, 14].

In this paper, we further investigate the Radon transform in the Clifford analysis setting. Some initial results can be found in [19, 20, 23]. More recently, other associated transforms such as the Segal-Bargmann, the Szegö-Radon and the Bargmann-Radon transforms have been investigated, see e.g. [7, 8, 17].

In our work, we continue the study of the Bargmann-Radon transform. This transform is defined as the projection of the real Bargmann module (of monogenic square integrable functions with Gaussian density) on the closed submodule of monogenic plane waves $\langle\underline{x}, \underline{\tau}\rangle^{\ell} \underline{\tau}$, where $\underline{\tau}=\underline{t}+i \underline{s}, \underline{t} \perp \underline{s}$. A complete characterization of this projection in the general monogenic setting can be found in [8].

Our main goal is to study the action of the Bargmann-Radon transform on a specific type of monogenic functions, namely axially monogenic functions. These are null solutions of the Dirac operator with an additional axial symmetry, modelled by a Vekua-type system, e.g. $[10,12,15,22,25]$. This theory is closely related to holomorphic functions of a single complex variable and the plane elliptic system, see $[6,21]$. Some preliminary work has already been done in [7] where the authors obtained the Cauchy-Kowalewski extension and inversion formula for of axially monogenic functions.

This paper is organized as follows. In Section 2, we introduce preliminary results related to Clifford analysis, in particular to Bargmann-Radon transform. In Section 3, we introduce a certain type of monogenic functions called axially monogenic functions. We compute the

${ }^{*}$ Corresponding author Ren Hu (E-mail: Ren.Hu@UGent.be) is supported by China Scholarship Council. 
Bargmann-Radon transform for axially monogenic functions by using the Funk-Hecke Theorem [13]. We also give an explicit formula for the transform in terms of hypergeometric functions $[2,16]$. In Section 4, we introduce the Cauchy-Kowalewski extension $[4,10]$ and give an explicit formula for the general $\mathrm{C}-\mathrm{K}$ extension. Finally we make use of the C-K extension in an example of the Bargmann-Radon transform for axially monogenic functions.

\section{Preliminary results}

The real Clifford algebra $\mathbb{R}_{m}$ is generated by the standard basis $\left\{\underline{e}_{1}, \underline{e}_{2}, \ldots, \underline{e}_{m}\right\}$ of $\mathbb{R}^{m}$ where the multiplication is defined by the relations $\underline{e}_{i} \underline{e}_{j}+\underline{e}_{j} \underline{e}_{i}=-2 \delta_{i j}$. A general element of $\mathbb{R}_{m}$ can be written as $a=\sum_{A} e_{A} a_{A}$, where $A=\left\{i_{1}, \ldots, i_{r}\right\} \subset\{1,2, \ldots, m\}, i_{1}<\ldots<i_{r}$ is a multi-index, $e_{A}=\underline{e}_{i_{1}} \underline{e}_{i_{2}} \ldots \underline{e}_{i_{r}}$ and $\underline{e}_{\varnothing}=1, a_{A} \in \mathbb{R}$. In particular, vectors in $\mathbb{R}^{m}$ are of the form $\underline{x}=\sum_{i=1}^{m} \underline{e}_{i} x_{i}$ which are equipped with the inner product $\langle\underline{x}, \underline{y}\rangle=\sum_{i=1}^{m} x_{i} y_{i}$ and the norm $|\underline{x}|=\left(\sum_{i=1}^{m} x_{i}^{2}\right)^{1 / 2}$.

We can also define the complex Clifford algebra by $\mathbb{C}_{m}=\mathbb{C} \otimes \mathbb{R}_{m}$ whose elements are of the form $c=\sum_{A} e_{A} c_{A}$, where $c_{A} \in \mathbb{C}$. Moreover, the Hermitian conjugation is then defined as

$$
(\lambda \mu)^{\dagger}=\mu^{\dagger} \lambda^{\dagger}, \quad\left(\mu_{A} \underline{e}_{A}\right)^{\dagger}=\mu_{A}^{c} \underline{e}_{A}^{\dagger}, \quad \underline{e}_{j}^{\dagger}=-\underline{e}_{j}, \quad j=1, \ldots, m, \quad \lambda, \mu \in \mathbb{C}_{m}
$$

where $\mu_{A}^{c}$ stands for the complex conjugate of the complex number $\mu_{A}$.

Definition 1 (Monogenic function). A function $f(\underline{x})$ is called (left-)monogenic on an open subset of $\mathbb{R}^{m}$ if it is differentiable and

$$
\partial_{\underline{x}} f(\underline{x})=0
$$

where $\partial_{\underline{x}}=\sum_{i=1}^{m} \underline{e}_{i} \partial_{x_{i}}$ is the Dirac operator.

Definition 2 (Harmonic functions). Any function $f: \mathbb{R}^{m} \rightarrow \mathbb{R}_{m}$ that satisfies the relation

$$
\Delta_{\underline{x}} f(\underline{x})=0
$$

is called a harmonic function, where $\Delta_{\underline{x}}$ is the Laplacian operator $\Delta_{\underline{x}}=-\partial_{\underline{x}}^{2}=\sum_{j=1}^{m} \partial_{x_{j}}^{2}$.

Remark 1. A polynomial $M_{k}(\underline{x})$ is called inner spherical monogenics of degree $k$ if

$$
\partial_{\underline{x}} M_{k}(\underline{x})=0, \quad \mathbb{E} M_{k}(\underline{x})=k M_{k}(\underline{x})
$$

where $\mathbb{E}=\sum_{j=1}^{m} x_{j} \partial_{x_{j}}$ is the Euler operator. Similarly, a polynomial $H_{k}(\underline{x})$ is called spherical harmonics of degree $k$ if

$$
\Delta_{\underline{x}} H_{k}(\underline{x})=0, \quad \mathbb{E} H_{k}(\underline{x})=k H_{k}(\underline{x}) .
$$

Let $B(0,1)$ be the unit ball $\left\{\underline{x} \in \mathbb{R}^{m}:|\underline{x}| \leq 1\right\}$ in $\mathbb{R}^{m}$ and $\mathbb{S}^{m-1}$ be its boundary, the unit sphere.

Definition 3. Let $\mathcal{M L}^{2}(B(0,1))$ be the right $\mathbb{C}_{m}$-module of monogenic functions, $f: B(0,1) \rightarrow$ $\mathbb{C}_{m}$ for which the restriction to unit sphere is square integrable, i.e.

$$
\left[\int_{\mathbb{S}^{m-1}} f^{\dagger}(\underline{\omega}) f(\underline{\omega}) d S(\underline{\omega})\right]_{0}<\infty
$$

The projection operator $[.]_{0}$ maps an element $a \in \mathbb{C}_{m}$ to its scalar-valued part which in this case is a positive real number. 
To study the Bargmann-Radon transform, it is necessary to introduce the real and the complex monogenic Bargmann module. Denote by $\mathcal{L}^{2}\left(\mathbb{R}^{m}\right)$ the space of Clifford-valued square integrable functions on $\mathbb{R}^{m}$.

Definition 4 (Monogenic Bargmann module). Denote the monogenic Bargmann module by $\mathcal{M B}\left(\mathbb{R}^{m}\right)$ which consists of monogenic functions $f$ on $\mathbb{R}^{m}$ such that

$$
f(\underline{x}) e^{-|\underline{x}|^{2} / 4} \in \mathcal{L}^{2}\left(\mathbb{R}^{m}\right) .
$$

This module is equipped with the $\mathbb{C}_{m}$-valued inner product

$$
\langle f, g\rangle_{\mathcal{M B}}=\frac{1}{(2 \pi)^{m / 2}} \int_{\mathbb{R}^{m}} e^{-|\underline{x}|^{2} / 2} f^{\dagger}(\underline{x}) g(\underline{x}) d \underline{x} .
$$

Remark 2. These concepts are usually introduced in the setting of several complex variables.

Let $\underline{z}=\underline{x}+i \underline{y}$ where $\underline{x}, \underline{y} \in \mathbb{R}^{m}$, i.e. $\underline{z}=\sum_{j=1}^{m} \underline{e}_{j} z_{j}$, where $z_{j}=x_{j}+i y_{j} \in \mathbb{C}$. Denote by $\mathcal{M}\left(\mathbb{C}^{m}\right)$ the right module of entire holomorphic functions $f(\underline{z})$ on $\mathbb{C}^{m}$ which are complex monogenic, i.e. $\partial_{\underline{z}} f(\underline{z})=0$, where $\partial_{\underline{z}}=\sum_{j=1}^{m} \underline{e}_{j} \partial_{z_{j}}, \partial_{z_{j}}=\frac{1}{2}\left(\partial_{x_{j}}-i \partial_{y_{j}}\right)$.

The Segal-Bargmann-Fock space $\mathcal{B}\left(\mathbb{C}^{m}\right)$ is the Hilbert module of $\mathbb{C}_{m}$-valued holomorphic entire functions in $\mathbb{C}^{m}$ which are square-integrable with respect to the $2 m$-dimensional Gaussian density, i.e.

$$
\frac{1}{\pi^{m}} \int_{\mathbb{C}^{m}} e^{-|\underline{z}|^{2}}|f(\underline{z})|^{2} d \underline{x} d \underline{y}<\infty
$$

This space is equipped with the inner product

$$
\langle f, g\rangle_{\mathcal{B}}=\frac{1}{\pi^{m}} \int_{\mathbb{C}^{m}} e^{-|\underline{z}|^{2}} f^{\dagger}(\underline{z}) g(\underline{z}) d \underline{x} d \underline{y} .
$$

Thus we can define the monogenic Bargmann module $\mathcal{M B}\left(\mathbb{C}^{m}\right)=\mathcal{M}\left(\mathbb{C}^{m}\right) \cap \mathcal{B}\left(\mathbb{C}^{m}\right)$ which is equipped with the same inner product as in (2).

Definition 5. For any given $\underline{\tau}=\underline{t}+i \underline{s}, \underline{t}, \underline{s} \in \mathbb{R}^{m}$, where $|\underline{t}|=|\underline{s}|=1$ and $\underline{t} \perp \underline{s}$, denote by $\mathcal{M B}(\underline{\tau})$ the closure of the right $\mathbb{C}_{m}$-module which consists of all finite linear combinations of $\langle\underline{x}, \underline{\tau}\rangle^{\ell} \underline{\tau}, \ell \in \mathbb{N}$, where $\langle\underline{x}, \underline{\tau}\rangle=\langle\underline{x}, \underline{t}\rangle+i\langle\underline{x}, \underline{s}\rangle$.

In the following, we introduce the Bargmann-Radon kernel and the Bargmann-Radon transform, more details can be found in [7, 8].

Definition 6 (Bargmann-Radon kernel associated with $\underline{\tau}$ ). Let $\underline{x}, \underline{y} \in \mathbb{R}^{m}$. For any given $\underline{\tau}=\underline{t}+i \underline{s}, \underline{t}, \underline{s} \in \mathbb{R}^{m}$, where $|\underline{t}|=|\underline{s}|=1$ and $\underline{t} \perp \underline{s}$, the Bargmann-Radon kernel $B_{\underline{\tau}}(\underline{x}, \underline{y})$ is defined as

$$
B_{\underline{\tau}}(\underline{x}, \underline{y})=\frac{\tau}{4} e^{\dagger} e^{-\frac{1}{2}\langle\underline{x}, \underline{\tau}\rangle\left\langle\underline{y}, \underline{\tau}^{\dagger}\right\rangle}
$$

or

$$
B_{\underline{\tau}}(\underline{x}, \underline{y})=\sum_{\ell=0}^{\infty} \gamma_{\ell}\langle\underline{x}, \underline{\tau}\rangle^{\ell} \underline{\tau} \underline{\tau}^{\dagger}\left\langle\underline{y}, \underline{\tau}^{\dagger}\right\rangle^{\ell}
$$

where $\gamma_{\ell}=\frac{(-1)^{\ell}}{\ell ! \cdot 2^{\ell+2}}$.

Remark 3. The Bargmann-Radon kernel $B_{\underline{\tau}}(\underline{x}, \underline{y})$ is a reproducing kernel for the $\mathbb{C}_{m}$-module $\mathcal{M B}(\underline{\tau})$, i.e. $f(\underline{y})=\left\langle B_{\underline{\tau}}(\underline{x}, \underline{y}), f(\underline{x})\right\rangle_{\mathcal{M B}}$ and it is Hermitian, i.e. $B_{\underline{\tau}}(\underline{y}, \underline{x})=B_{\underline{\tau}}(\underline{x}, \underline{y})^{\dagger}$.

Definition 7 (Bargmann-Radon transform). The Bargmann-Radon transform is a projection operator from $\mathcal{M B}\left(\mathbb{R}^{m}\right)$ to $\mathcal{M B}(\underline{\tau})$, which can be written as the integral

$$
R_{\underline{\tau}}[f](\underline{x})=\frac{1}{(2 \pi)^{m / 2}} \int_{\mathbb{R}^{m}} e^{-|\underline{y}|^{2} / 2} B_{\underline{\tau}}(\underline{x}, \underline{y}) f(\underline{y}) d \underline{y} .
$$


Funk-Hecke theorem is essential for our computation. By using the previous notations, the theorem can be stated as follows (see $[1,13])$.

Theorem 1 (Funk-Hecke theorem). Let $\xi, \eta \in \mathbb{S}^{m-1}$ and let $\psi$ be a real-valued function whose domain contains $[-1,1]$. Let $H_{k}(\xi)$ be a spherical harmonic polynomial of degree $k$. Then we have

$$
\int_{\mathbb{S}^{m-1}} \psi(\langle\underline{\xi}, \underline{\eta}\rangle) H_{k}(\underline{\eta}) d S(\underline{\eta})=\frac{k ! A_{m-1}}{(m-2)_{k}} H_{k}(\underline{\xi}) \int_{-1}^{1} \psi(t) C_{k}^{\frac{m}{2}-1}(t)\left(1-t^{2}\right)^{(m-3) / 2} d t .
$$

where $d S(\underline{\eta})$ is the scalar element of surface area on $\mathbb{S}^{m-1} . C_{k}^{\alpha}(t)$ is a Gegenbauer polynomial, $A_{m}=\frac{2 \pi^{m / 2}}{\Gamma\left(\frac{m}{2}\right)}$ is the area of the unit sphere in $\mathbb{R}^{m}$ and $(a)_{k}=a(a+1) \cdots(a+k-1)$ is the Pochhammer symbol.

To be able to calculate the integral on the right hand side of (6), we need the following lemma (see [16]).

Lemma 1. Let $C_{k}^{\alpha}(t)$ be a Gegenbauer polynomial, then we have

$$
\int_{-1}^{1} t^{j} C_{k}^{\alpha}(t)\left(1-t^{2}\right)^{\alpha-\frac{1}{2}} d t=L(k, \alpha, j)
$$

where

$$
L(k, \alpha, j)=\left((-1)^{j+k}+1\right) \frac{\pi 2^{-2 \alpha-j} \Gamma(k+2 \alpha) \Gamma(j+1)}{k ! \Gamma(\alpha) \Gamma\left(1+\frac{k}{2}+\alpha+\frac{j}{2}\right) \Gamma\left(1+\frac{j}{2}-\frac{k}{2}\right)}
$$

for all $j \in \mathbb{N} \cup\{0\}$.

\section{Bargmann-Radon transform for axially monogenic functions}

In this section we first introduce axially monogenic functions. Then we compute the BargmannRadon transform for axially monogenic functions and give explicit formulas for the transform.

Let $\Omega$ be an open subset of $\mathbb{R}^{m}$ which is invariant under $S O(m-1)$. According to $[21,22]$, any left monogenic function $f(\underline{x})$ in $\Omega$ can be written as

$$
f(\underline{x})=\sum_{k=0}^{\infty} \Pi_{k} f(\underline{x}),
$$

where $\Pi_{k} f(\underline{x})$ is a so-called axial monogenic function of degree $k$. Let $\left(x_{1}, \rho, \eta\right) \in \mathbb{R} \times \mathbb{R}_{+} \times \mathbb{S}^{m-2}$ be cylindrical coordinates such that $\underline{x}=x_{1} \underline{e}_{1}+\rho \underline{\eta}$. Then for a fixed pair $\left(\bar{x}_{1}, \rho\right)$, the function $\Pi_{k} f(\underline{x})$ has the form

$$
\Pi_{k} f(\underline{x})=\left(A\left(x_{1}, \rho\right) \underline{e}_{1}+\underline{\eta} B\left(x_{1}, \rho\right)\right) M_{k}(\underline{\eta}),
$$

or equivalently

$$
\Pi_{k} f(\underline{x})=\left(A\left(x_{1}, \rho\right)+\underline{e}_{1} \underline{\eta} B\left(x_{1}, \rho\right)\right) M_{k}(\underline{\eta}),
$$

where $M_{k}(\underline{\eta})$ is inner spherical monogenic polynomial of degree $k$ on $\mathbb{S}^{m-2}$. Moreover, the functions $\bar{A}$ and $B$ satisfy the Vekua-type system (see [22]),

$$
\left\{\begin{array}{l}
\frac{\partial}{\partial x_{1}} A\left(x_{1}, \rho\right)-\frac{\partial}{\partial \rho} B\left(x_{1}, \rho\right)=\frac{k+m-2}{\rho} B\left(x_{1}, \rho\right), \\
\frac{\partial}{\partial x_{1}} B\left(x_{1}, \rho\right)+\frac{\partial}{\partial \rho} A\left(x_{1}, \rho\right)=\frac{k}{\rho} A\left(x_{1}, \rho\right) .
\end{array}\right.
$$

Functions of the form (9) and (10) are called axially monogenic functions, see e.g. [10, 12, $15,22,25]$. For the sake of simplicity, we will project axially monogenic function of the form (9) on the submodule $\mathcal{M B}(\underline{\tau})$ with $\underline{t}=\underline{e}_{1}$ and $\underline{s} \in \mathbb{S}^{m-2}$. The other case can be obtained in a similar way. 
Theorem 2. The Bargmann-Radon transform of an axially monogenic function $f(y)$ of the form (9) can be written as

$$
R_{\underline{\tau}}[f](\underline{x})=\underline{\tau} \underline{\tau}^{\dagger}\left(E(\langle\underline{x}, \underline{\tau}\rangle) \underline{e}_{1}+F(\langle\underline{x}, \underline{\tau}\rangle) \underline{s}\right) M_{k}(\underline{s})
$$

where $E$ and $F$ are holomorphic functions of the variable $\langle\underline{x}, \underline{\tau}\rangle, M_{k}$ is spherical monogenic of degree $k$.

Proof. Let $\left(y_{1}, \rho, \underline{\eta}\right) \in \mathbb{R} \times \mathbb{R}_{+} \times \mathbb{S}^{m-2}$ be cylindrical coordinates such that $\underline{y}=y_{1} \underline{e}_{1}+\rho \underline{\eta}$. Since $\underline{\tau}=\underline{t}+i \underline{s}, \underline{t}=\underline{e}_{1}, \underline{s} \in \mathbb{S}^{m-2}, \underline{t} \perp \underline{s}$, we have

$$
\left\langle\underline{y}, \underline{\tau}^{\dagger}\right\rangle=\left\langle y_{1} \underline{e}_{1}+\rho \underline{\eta},-\underline{t}+i \underline{s}\right\rangle=-y_{1}+i \rho\langle\underline{\eta}, \underline{s}\rangle .
$$

Therefore Bargmann-Radon kernel (3) can be written as

$$
B_{\underline{\tau}}(\underline{x}, \underline{y})=\frac{\underline{\tau} \underline{\tau}^{\dagger}}{4} e^{-\frac{1}{2}\langle\underline{x}, \underline{\tau}\rangle\left(-y_{1}+i \rho\langle\underline{\eta}, \underline{s}\rangle\right)}=\underline{\tau} \underline{\tau}^{\dagger} \mathcal{B}\left(\langle\underline{x}, \underline{\tau}\rangle,-y_{1}+i \rho\langle\underline{\eta}, \underline{s}\rangle\right),
$$

where $\mathcal{B}\left(\langle\underline{x}, \underline{\tau}\rangle,-y_{1}+i \rho\langle\underline{\eta}, \underline{s}\rangle\right)=\frac{1}{4} e^{-\frac{1}{2}\langle\underline{x}, \underline{\tau}\rangle\left(-y_{1}+i \rho\langle\underline{\eta}, \underline{s}\rangle\right)}$. By using cylindrical coordinates, the Bargmann-Radon transform (5) can be expressed as

$$
R_{\underline{\tau}}[f](\underline{x})=\frac{1}{(2 \pi)^{m / 2}} \int_{\mathbb{R}} \int_{0}^{+\infty} \int_{\mathbb{S}^{m-2}} e^{-\frac{y_{1}^{2}+\rho^{2}}{2}} B_{\underline{\tau}}\left(\underline{x}, y_{1} \underline{e}_{1}+\rho \underline{\eta}\right) f\left(y_{1}, \rho, \underline{\eta}\right) \rho^{m-2} d S(\underline{\eta}) d \rho d y_{1} .
$$

In order to further compute the transform, we substitute the function $f$ by its axially monogenic form (9). Then we obtain

$$
\begin{aligned}
R_{\underline{\tau}}[f](\underline{x})=\frac{\underline{\tau} \underline{\tau}^{\dagger}}{(2 \pi)^{m / 2}} \int_{\mathbb{R}} \int_{0}^{+\infty} & \int_{\mathbb{S}^{m-2}} e^{-\frac{y_{1}^{2}+\rho^{2}}{2}} \mathcal{B}\left(\langle\underline{x}, \underline{\tau}\rangle,-y_{1}+i \rho\langle\underline{\eta}, \underline{s}\rangle\right) \\
& \times\left(A\left(y_{1}, \rho\right) \underline{e}_{1}+B\left(y_{1}, \rho\right) \underline{\eta}\right) M_{k}(\underline{\eta}) \rho^{m-2} d S(\underline{\eta}) d \rho d y_{1} .
\end{aligned}
$$

We first consider the spherical integral in $R_{\underline{\tau}}[f](\underline{x})$, i.e.

$$
I=\int_{\mathbb{S}^{m-2}} e^{-\frac{y_{1}^{2}+\rho^{2}}{2}} \mathcal{B}\left(\langle\underline{x}, \underline{\tau}\rangle,-y_{1}+i \rho\langle\underline{\eta}, \underline{s}\rangle\right)\left(A\left(y_{1}, \rho\right) \underline{e}_{1}+B\left(y_{1}, \rho\right) \underline{\eta}\right) M_{k}(\underline{\eta}) d S(\underline{\eta}) .
$$

It is natural to split the above integral into two parts

$$
I_{1}=\int_{\mathbb{S}^{m-2}} e^{-\frac{y_{1}^{2}+\rho^{2}}{2}} \mathcal{B}\left(\langle\underline{x}, \underline{\tau}\rangle,-y_{1}+i \rho\langle\underline{\eta}, \underline{s}\rangle\right) A\left(y_{1}, \rho\right) \underline{e}_{1} M_{k}(\underline{\eta}) d S(\underline{\eta})
$$

and

$$
I_{2}=\int_{\mathbb{S}^{m-2}} e^{-\frac{y_{1}^{2}+\rho^{2}}{2}} \mathcal{B}\left(\langle\underline{x}, \underline{\tau}\rangle,-y_{1}+i \rho\langle\underline{\eta}, \underline{s}\rangle\right) B\left(y_{1}, \rho\right) \underline{\eta} M_{k}(\underline{\eta}) d S(\underline{\eta}) .
$$

Applying Funk-Hecke theorem on $I_{1}$ results in

$$
I_{1}=\frac{k ! A_{m-2}}{(m-3)_{k}} \int_{-1}^{1} \mathcal{B}\left(\langle\underline{x}, \underline{\tau}\rangle,-y_{1}+i \rho t\right) C_{k}^{\frac{m-3}{2}}\left(1-t^{2}\right)^{\frac{m-4}{2}} d t\left(e^{-\frac{y_{1}^{2}+\rho^{2}}{2}} A\left(y_{1}, \rho\right) \underline{e}_{1} M_{k}(\underline{s})\right) .
$$

Similarly, we obtain

$$
I_{2}=\frac{(k+1) ! A_{m-2}}{(m-3)_{k+1}} \int_{-1}^{1} \mathcal{B}\left(\langle\underline{x}, \underline{\tau}\rangle,-y_{1}+i \rho t\right) C_{k+1}^{\frac{m-3}{2}}\left(1-t^{2}\right)^{\frac{m-4}{2}} d t\left(e^{-\frac{y_{1}^{2}+\rho^{2}}{2}} B\left(y_{1}, \rho\right) \underline{s} M_{k}(\underline{s})\right) .
$$


Therefore, the transform can be written as

$$
R_{\underline{\tau}}[f](\underline{x})=\underline{\tau} \underline{\tau}^{\dagger}\left(E(\langle\underline{x}, \underline{\tau}\rangle) \underline{e}_{1}+F(\langle\underline{x}, \underline{\tau}\rangle) \underline{s}\right) M_{k}(\underline{s})
$$

in which

$E(\langle\underline{x}, \underline{\tau}\rangle)=\phi_{k} \int_{\mathbb{R}} \int_{0}^{+\infty} \int_{-1}^{1} e^{-\frac{y_{1}^{2}+\rho^{2}}{2}} \mathcal{B}\left(\langle\underline{x}, \underline{\tau}\rangle,-y_{1}+i \rho t\right) C_{k}^{\frac{m-3}{2}}\left(1-t^{2}\right)^{\frac{m-4}{2}} A\left(y_{1}, \rho\right) \rho^{m-2} d t d \rho d y_{1}$,

and

$F(\langle\underline{x}, \underline{\tau}\rangle)=\phi_{k+1} \int_{\mathbb{R}} \int_{0}^{+\infty} \int_{-1}^{1} e^{-\frac{y_{1}^{2}+\rho^{2}}{2}} \mathcal{B}\left(\langle\underline{x}, \underline{\tau}\rangle,-y_{1}+i \rho t\right) C_{k+1}^{\frac{m-3}{2}}\left(1-t^{2}\right)^{\frac{m-4}{2}} B\left(y_{1}, \rho\right) \rho^{m-2} d t d \rho d y_{1}$

where $\phi_{k}=\frac{k !}{\pi \Gamma\left(\frac{m-2}{2}\right)(m-3)_{k}}$ is a scalar coefficient. Since the Bargmann-Radon kernel $B_{\underline{\tau}}(\underline{x}, \underline{y})$ is holomorphic, therefore functions $E$ and $F$ are also holomorphic of the variable $\langle\underline{x}, \underline{\tau}\rangle$.

In the following, we investigate the explicit form of the Bargmann-Radon transform for axially monogenic functions. We first compute the integrals (11) and (12). Then we try to present the result in a concrete form.

Similar to [8], the Bargmann-Radon kernel can also be written as in (4), then we have

$$
\mathcal{B}\left(\langle\underline{x}, \underline{\tau}\rangle,-y_{1}+i \rho\langle\underline{\eta}, \underline{s}\rangle\right)=\sum_{\ell=0}^{\infty} \gamma_{\ell}\langle\underline{x}, \underline{\tau}\rangle^{\ell}\left(-y_{1}+i \rho\langle\underline{\eta}, \underline{s}\rangle\right)^{\ell} .
$$

First we consider $I_{1}$ again in (11). Replacing the kernel in $I_{1}$ results in

$$
I_{1}=\frac{k ! A_{m-2}}{(m-3)_{k}} \int_{-1}^{1} \sum_{\ell=0}^{\infty} \gamma_{\ell}\langle\underline{x}, \underline{\tau}\rangle^{\ell}\left(-y_{1}+i \rho t\right)^{\ell} C_{k}^{\frac{m-3}{2}}\left(1-t^{2}\right)^{\frac{m-4}{2}} d t\left(e^{-\frac{y_{1}^{2}+\rho^{2}}{2}} A\left(y_{1}, \rho\right) \underline{e}_{1} M_{k}(\underline{s})\right)
$$

Using the binomial formula to compute $\left(-y_{1}+i \rho t\right)^{\ell}$ gives

$I_{1}=\sum_{\ell=0}^{\infty} \sum_{j=0}^{\ell} \gamma_{\ell}\langle\underline{x}, \underline{\tau}\rangle^{\ell} \frac{k ! A_{m-2}}{(m-3)_{k}}\left(\begin{array}{l}\ell \\ j\end{array}\right)\left(-y_{1}\right)^{\ell-j}(i \rho)^{j} \int_{-1}^{1} t^{j} C_{k}^{\frac{m-3}{2}}\left(1-t^{2}\right)^{\frac{m-4}{2}} d t\left(e^{-\frac{y_{1}^{2}+\rho^{2}}{2}} A\left(y_{1}, \rho\right) \underline{e}_{1} M_{k}(\underline{s})\right)$.

Making use of formula (7) to compute the integral results in

$$
I_{1}=\sum_{j=0}^{\infty} \sum_{\ell=0}^{\infty} \gamma_{\ell+j}\langle\underline{x}, \underline{\tau}\rangle^{\ell+j} \frac{k ! A_{m-2}}{(m-3)_{k}}\left(\begin{array}{c}
\ell+j \\
j
\end{array}\right)\left(-y_{1}\right)^{\ell}(i \rho)^{j} L\left(k, \frac{m-3}{2}, j\right) e^{-\frac{y_{1}^{2}+\rho^{2}}{2}} A\left(y_{1}, \rho\right) \underline{e}_{1} M_{k}(\underline{s})
$$

where $L(n, \alpha, j)$ is defined in (8). Denote $\lambda=\langle\underline{x}, \underline{\tau}\rangle, I_{1}$ can be written as

$$
\begin{aligned}
I_{1}=\frac{\pi^{\frac{m-1}{2}}}{16} & \left(\frac{4\left((-1)^{k}+1\right)}{\Gamma\left(\frac{2-k}{2}\right) \Gamma\left(\frac{k+m-1}{2}\right)}{ }_{1} F_{2}\left(1 ; \frac{2-k}{2}, \frac{k+m-1}{2} ;-\frac{\rho^{2} \lambda^{2}}{16}\right)\right. \\
& \left.+i \frac{\left((-1)^{k}-1\right) \rho \lambda}{\Gamma\left(\frac{3-k}{2}\right) \Gamma\left(\frac{k+m}{2}\right)}{ }_{1} F_{2}\left(1 ; \frac{3-k}{2}, \frac{k+m}{2} ;-\frac{\rho^{2} \lambda^{2}}{16}\right)\right) e^{-\frac{y_{1}^{2}+\rho^{2}-y_{1} \lambda}{2}} A\left(y_{1}, \rho\right) \underline{e}_{1} M_{k}(\underline{s}),
\end{aligned}
$$

where ${ }_{1} F_{2}\left(a_{1} ; b_{1}, b_{2} ; z\right)$ is the hypergeometric function defined in e.g. [16]. Let us consider the scalar function

$$
C_{m, k}(\lambda, u, v)=\frac{1}{8} \frac{\pi^{\frac{m-1}{2}}}{\Gamma(u) \Gamma(v)} e^{-\frac{y_{1}^{2}+\rho^{2}-y_{1} \lambda}{2}}{ }_{1} F_{2}\left(1 ; u, v ;-\frac{\rho^{2} \lambda^{2}}{16}\right),
$$


then $I_{1}$ can be written as

$$
I_{1}= \begin{cases}-i \rho \lambda C_{m, k}\left(\lambda, \frac{3-k}{2}, \frac{m+k}{2}\right) A\left(y_{1}, \rho\right) \underline{e}_{1} M_{k}(\underline{s}) & k \text { is odd } \\ 4 C_{m, k}\left(\lambda, \frac{2-k}{2}, \frac{m+k-1}{2}\right) A\left(y_{1}, \rho\right) \underline{e}_{1} M_{k}(\underline{s}) & k \text { is even. }\end{cases}
$$

Following a similar procedure, we can have

$$
I_{2}= \begin{cases}4 C_{m, k}\left(\lambda, \frac{1-k}{2}, \frac{m+k}{2}\right) B\left(y_{1}, \rho\right) \underline{s} M_{k}(\underline{s}) & k \text { is odd } \\ i \rho \lambda C_{m, k}\left(\lambda, \frac{2-k}{2}, \frac{m+k+1}{2}\right) B\left(y_{1}, \rho\right) \underline{s} M_{k}(\underline{s}) & k \text { is even. }\end{cases}
$$

Therefore we obtain the following theorem.

Theorem 3. The Bargmann-Radon transform for axially monogenic functions can be written in the form

$$
R_{\underline{\tau}}[f](\underline{x})= \begin{cases} & \frac{\tau}{(2 \pi)^{\dagger / 2}} \int_{\mathbb{R}} \int_{0}^{+\infty}\left(-i \rho^{m-1} \lambda C_{m, k}\left(\lambda, \frac{3-k}{2}, \frac{m+k}{2}\right) A\left(y_{1}, \rho\right) \underline{e}_{1}\right. \\ & \left.+4 \rho^{m-2} C_{m, k}\left(\lambda, \frac{1-k}{2}, \frac{m+k}{2}\right) B\left(y_{1}, \rho\right) \underline{s}\right) M_{k}(\underline{s}) d \rho d y_{1}, \quad k \text { is odd, } \\ & \frac{\tau}{(2 \pi)^{m / 2}} \int_{\mathbb{R}} \int_{0}^{+\infty}\left(4 \rho^{m-2} C_{m, k}\left(\lambda, \frac{2-k}{2}, \frac{m+k-1}{2}\right) A\left(y_{1}, \rho\right) \underline{e}_{1}\right. \\ & \left.+i \rho^{m-1} \lambda C_{m, k}\left(\lambda, \frac{2-k}{2}, \frac{m+k+1}{2}\right) B\left(y_{1}, \rho\right) \underline{s}\right) M_{k}(\underline{s}) d \rho d y_{1}, \quad k \text { is even. }\end{cases}
$$

where $k$ is the degree of homogeneity of monogenic homogeneous polynomial $M_{k}(\underline{s})$.

\section{Bargmann-Radon transform for the C-K extension}

In this section, we compute the Bargmann-Radon transform of the C-K extension of analytic function $g(\rho \eta)$ defined in a radially symmetric domain. In addition, we apply these results to the case when $g(\rho \underline{\eta})=(\rho \underline{\eta})^{s} M_{k}(\rho \underline{\eta})$.

In general, every monogenic function $f(\underline{x})$ is determined by its restriction $f(0, \underline{v})$ to the hyperplane $x_{1}=0$, where $\underline{x}=x_{1} \underline{e}_{1}+\underline{v}, \underline{v} \in \mathbb{R}^{m-1}$. Conversely, any given real analytic function $g(\underline{v})$ has a monogenic extension, which is the so-called $\mathrm{C}-\mathrm{K}$ extension and reads as

$$
f(\underline{x})=C K(g)(\underline{x})=e^{-x_{1} \underline{e}_{1} \partial_{\underline{v}}} g(\underline{v})
$$

where $g(\underline{v})=\left.f(\underline{x})\right|_{x_{1}=0}$, see more in $[10,22]$. Therefore, we have

$$
C K(g)(\underline{x})=\sum_{\ell=0}^{\infty} \frac{\left(x_{1} \underline{e}_{1} \partial_{\underline{v}}\right)^{\ell}}{\ell !} g(\underline{v}) .
$$

In the following, we compute the Bargmann-Radon transform for axially monogenic functions which are obtained via the $\mathrm{C}-\mathrm{K}$ extension of the function

$$
g(\underline{v})=\left(a\left(|\underline{v}|^{2}\right) \underline{e}_{1}+\underline{v} b\left(|\underline{v}|^{2}\right)\right) M_{k}(\underline{v})
$$

where the polynomial $M_{k}(\underline{v})$ is monogenic and homogeneous of degree $k$.

Remark 4. Any real analytic function in a radially symmetric domain can be decomposed into a series of functions of the form (15). Therefore, it is sufficient for us to consider $g(\underline{v})$ in the following computation.

Theorem 4. The $C-K$ extension of $g(\underline{v})$ of the form (15) can be written as

$$
f(\underline{x})=\left(A\left(x_{1},|\underline{v}|^{2}\right) \underline{e}_{1}+B\left(x_{1},|\underline{v}|^{2}\right) \underline{v}\right) M_{k}(\underline{v})
$$

where $A$ and $B$ are scalar-valued functions. 
In order to find the explicit form of the functions $A$ and $B$ in (16), we substitute $g(\underline{v})$ of the form (15) in the C-K extension (14), i.e.

$$
C K(g)(\underline{x})=\sum_{\ell=0}^{\infty} \frac{\left(x_{1} \underline{e}_{1} \partial_{\underline{v}}\right)^{\ell}}{\ell !}\left(\underline{e}_{1} a\left(|\underline{v}|^{2}\right) M_{k}(\underline{v})\right)+\sum_{\ell=0}^{\infty} \frac{\left(x_{1} \underline{e}_{1} \partial_{\underline{v}}\right)^{\ell}}{\ell !}\left(\underline{v} b\left(|\underline{v}|^{2}\right) M_{k}(\underline{v})\right) .
$$

To compute the C-K extension, we need to investigate the actions $\partial_{v}^{\ell}\left(a\left(|\underline{v}|^{2}\right) M_{k}(\underline{v})\right)$ and $\partial_{\underline{v}}^{\ell}\left(\underline{v} b\left(|\underline{v}|^{2}\right) M_{k}(\underline{v})\right)$. To this end, we require the following lemmas.

Lemma 2. Let $n \in \mathbb{N}, \underline{x} \in \mathbb{R}_{m}$, then

$$
\partial_{\underline{x}} \underline{x}^{n}= \begin{cases}-n \underline{x}^{n-1}+\underline{x}^{n} \partial_{\underline{x}} & \text { if } n \text { is even } \\ -(m+n-1) \underline{x}^{n-1}-\underline{x}^{n} \partial_{\underline{x}}-2 \underline{x}^{n-1} \mathbb{E} & \text { if } n \text { is odd } .\end{cases}
$$

Lemma 3. For any given real-valued function $f\left(|\underline{x}|^{2}\right)$, we have

$\partial_{\underline{x}} \underline{x}^{n} f\left(|\underline{x}|^{2}\right)= \begin{cases}-n \underline{x}^{n-1} f\left(|\underline{x}|^{2}\right)+2 \underline{x}^{n+1} f^{\prime}\left(|\underline{x}|^{2}\right)+\underline{x}^{n} f\left(|\underline{x}|^{2}\right) \partial_{\underline{x}} & \text { if } n \text { is even, } \\ -(m+n-1) \underline{x}^{n-1} f\left(|\underline{x}|^{2}\right)+2 \underline{x}^{n+1} f^{\prime}\left(|\underline{x}|^{2}\right)-\underline{x}^{n} f\left(|\underline{x}|^{2}\right) \partial_{\underline{x}}-2 \underline{x}^{n-1} f\left(|\underline{x}|^{2}\right) \mathbb{E} & \text { if } n \text { is odd. }\end{cases}$

Proof. Denote by $P(\underline{x})$ any Clifford-valued polynomial. Consider even and odd cases separately with respect to $n$.

1. When $n$ is odd, we apply Lemma 2 and obtain

$$
\partial_{\underline{x}} \underline{x}^{n} f\left(|\underline{x}|^{2}\right) P(\underline{x})=\left(-(m+n-1) \underline{x}^{n-1}-\underline{x}^{n} \underline{\partial}_{\underline{x}}-2 \underline{x}^{n-1} \mathbb{E}\right) f\left(|\underline{x}|^{2}\right) P(\underline{x}) .
$$

By further computation of the Dirac operator $\partial_{\underline{x}}$ and the Euler operator $\mathbb{E}$ on the function $f\left(|\underline{x}|^{2}\right)$, we thus obtain

$$
\partial_{\underline{x}} \underline{x}^{n} f\left(|\underline{x}|^{2}\right) P(\underline{x})=\left(-(m+n-1) \underline{x}^{n-1} f\left(|\underline{x}|^{2}\right)+2 \underline{x}^{n+1} f^{\prime}\left(|\underline{x}|^{2}\right)-\underline{x}^{n} f\left(|\underline{x}|^{2}\right) \partial_{\underline{x}}-2 \underline{x}^{n-1} f\left(|\underline{x}|^{2}\right) \mathbb{E}\right) P(\underline{x}) .
$$

2. When $n$ is even, we apply Lemma 2 again

$$
\begin{aligned}
\partial_{\underline{x}} \underline{x}^{n} f\left(|\underline{x}|^{2}\right) P(\underline{x}) & =-n \underline{x}^{n-1} f\left(|\underline{x}|^{2}\right) P(\underline{x})+\underline{x}^{n} \partial_{\underline{x}} f\left(|\underline{x}|^{2}\right) P(\underline{x}) \\
& =\left(-n \underline{x}^{n-1} f\left(|\underline{x}|^{2}\right)+2 \underline{x}^{n+1} f^{\prime}\left(|\underline{x}|^{2}\right)+\underline{x}^{n} f\left(|\underline{x}|^{2}\right) \partial_{\underline{x}}\right) P(\underline{x}) .
\end{aligned}
$$

This proves the lemma.

Remark 5. Since $\underline{x}=x_{1} \underline{e}_{1}+\underline{v}$, with $\underline{v}=\sum_{i=2}^{m} \underline{e}_{i} x_{i}$. Let $\partial_{\underline{v}}=\sum_{i=2}^{m} \underline{e}_{i} \partial_{x_{i}}$ be the Dirac operator corresponding to $\underline{v}$. It is clear that $\underline{e}_{1}$ is orthogonal to $\underline{v}$, i.e. $\underline{e}_{1} \underline{v}=-\underline{v} \underline{e}_{1}$ and $\underline{e}_{1} \partial_{\underline{v}}=-\partial_{\underline{v}} \underline{e}_{1}$.

Now we consider the action of the operator $\underline{e}_{1} \partial_{\underline{v}}$ on $\underline{e}_{1} a\left(|\underline{v}|^{2}\right) M_{k}(\underline{v})$. We thus have the following lemma.

Lemma 4. Let $s=m+2 k, u=|\underline{v}|^{2}$. Let $M_{k}(\underline{v})$ be a monogenic homogeneous polynomial of degree $k$, then we have

$$
\left(\underline{e}_{1} \partial_{\underline{v}}\right)^{\ell} \underline{e}_{1} a(u) M_{k}(\underline{v})= \begin{cases}2^{2 j+1} \sum_{n=0}^{j} \alpha_{2 j+1, n} \underline{v}^{2 n+1} a^{(n+j+1)}(u) M_{k}(\underline{v}) & \ell=2 j+1, \\ 2^{2 j} \underline{e}_{1} \sum_{n=0}^{j} \alpha_{2 j, n} \underline{v}^{2 n} a^{(n+j)}(u) M_{k}(\underline{v}) & \ell=2 j,\end{cases}
$$

$j=0,1,2, \ldots$, where $\alpha_{2 j+1, n}=\frac{(-1)^{j+n}\left(\begin{array}{c}j \\ j-n\end{array}\right) \Gamma\left(j+\frac{s+1}{2}\right)}{\Gamma\left(n+\frac{s+1}{2}\right)}$ and $\alpha_{2 j, n}=\frac{(-1)^{j+n}\left(\begin{array}{c}j \\ j\end{array}\right) \Gamma\left(j+\frac{s-1}{2}\right)}{\Gamma\left(n+\frac{s-1}{2}\right)}$. 
Proof. We prove the Lemma by using induction on $\ell$.

1. For $\ell=1$, we easily compute

$$
\left(\underline{e}_{1} \partial_{\underline{v}}\right) \underline{e}_{1} a(u) M_{k}(\underline{v})=2 a^{\prime}(u) \underline{v} M_{k}(\underline{v}) .
$$

2. For $\ell=2$, we obtain from the previous case that

$$
\left(\underline{e}_{1} \partial_{\underline{v}}\right)^{2} \underline{e}_{1} a(u) M_{k}(\underline{v})=2 \underline{e}_{1}\left(-(s-1) a^{\prime}(u)+2 \underline{v}^{2} a^{\prime \prime}(u)\right) M_{k}(\underline{v}) .
$$

3. Suppose cases when $\ell<2 p$ are true. For $\ell=2 p$, using the induction hypothesis results in

$$
\left(\underline{e}_{1} \partial_{\underline{v}}\right)^{2 p} \underline{e}_{1} a(u) M_{k}(\underline{v})=\underline{e}_{1} \partial_{\underline{v}}\left(2^{2 p-1} \sum_{n=0}^{p-1} \frac{(-1)^{p+n-1}\left(\begin{array}{c}
p-1 \\
p-n-1
\end{array}\right) \Gamma\left(p+\frac{s-1}{2}\right)}{\Gamma\left(n+\frac{s+1}{2}\right)} \underline{v}^{2 n+1} a^{(n+p)}(u) M_{k}(\underline{v})\right) .
$$

By using Lemma 3 and the fact that $M_{k}(\underline{v})$ is monogenic, we have

$$
\left(\underline{e}_{1} \partial_{\underline{v}}\right)^{2 p} \underline{e}_{1} a(u) M_{k}(\underline{v})=2^{2 p} \underline{e}_{1} \sum_{n=0}^{p} \frac{(-1)^{p+n}\left(\begin{array}{c}
p \\
p-n
\end{array}\right) \Gamma\left(p+\frac{s-1}{2}\right)}{\Gamma\left(n+\frac{s-1}{2}\right)} \underline{v}^{2 n} a^{(n+p)}(u) M_{k}(\underline{v})
$$

which proves the result for $\ell=2 p$.

4. When $\ell=2 p+1$, we use the induction hypothesis again and obtain

$$
\left(\underline{e}_{1} \partial_{\underline{v}}\right)^{2 p+1} \underline{e}_{1} a(u) M_{k}(\underline{v})=\underline{e}_{1} \partial_{\underline{v}}\left(2^{2 p} \underline{e}_{1} \sum_{n=0}^{p} \frac{(-1)^{p+n}\left(\begin{array}{c}
p \\
p-n
\end{array}\right) \Gamma\left(p+\frac{s-1}{2}\right)}{\Gamma\left(n+\frac{s-1}{2}\right)} \underline{v}^{2 n} a^{(n+p)}(u) M_{k}(\underline{v})\right) .
$$

Similarly, by Lemma 3 we get

$$
\left(\underline{e}_{1} \partial_{\underline{v}}\right)^{2 p+1} \underline{e}_{1} a(u) M_{k}(\underline{v})=2^{2 p+1} \sum_{n=0}^{p} \frac{(-1)^{p+n}\left(\begin{array}{c}
p \\
p-n
\end{array}\right) \Gamma\left(p+\frac{s+1}{2}\right)}{\Gamma\left(n+\frac{s+1}{2}\right)} \underline{v}^{2 n+1} a^{(n+p+1)}(u) M_{k}(\underline{v}),
$$

which proves the result for $\ell=2 p+1$.

Remark 6. The coefficients $\alpha_{2 j+1, n}$ and $\alpha_{2 j, n}$ in the lemma have recurrence relations which are

$$
\left\{\begin{array}{l}
\alpha_{2 j+1, n}=-(n+1) \alpha_{2 j, n+1}+\alpha_{2 j, n} \\
2 \alpha_{2 j, n}=-(m+2 k+2 n-1) \alpha_{2 j-1, n}+2 \alpha_{2 j-1, n-1}
\end{array}\right.
$$

where $n=0,1,2, \ldots, j-1$.

Using the same method, we can obtain the following Lemma.

Lemma 5. For any given function $b(u)$, we have

$$
\begin{gathered}
\left(\underline{e}_{1} \partial_{\underline{v}}\right)^{\ell} \underline{v} b(u) M_{k}(\underline{v})= \begin{cases}2^{2 j+1} \underline{e}_{1} \sum_{n=0}^{j+1} \beta_{2 j+1, n} \underline{v}^{2 n} b^{(n+j)}(u) M_{k}(\underline{v}) & \ell=2 j+1, \\
2^{2 j} \sum_{n=0}^{j} \beta_{2 j, n} \underline{v}^{2 n+1} b^{(n+j)}(u) M_{k}(\underline{v}) & \ell=2 j,\end{cases} \\
j=0,1,2, \ldots, \text { where } \beta_{2 j+1, n}=\frac{(-1)^{j+n+1}\left(\begin{array}{c}
j+1 \\
j-n+1
\end{array}\right) \Gamma\left(j+\frac{s+1}{2}\right)}{\Gamma\left(n+\frac{s-1}{2}\right)} \text { and } \beta_{2 j, n}=\frac{(-1)^{j+n}\left(\begin{array}{c}
j \\
j-n
\end{array}\right) \Gamma\left(j+\frac{s+1}{2}\right)}{\Gamma\left(n+\frac{s+1}{2}\right)} .
\end{gathered}
$$


Remark 7. The coefficients $\beta_{2 j+1, n}$ and $\beta_{2 j, n}$ also satisfy the recurrence relations

$$
\left\{\begin{array}{l}
2 \beta_{2 j+1, n}=-(s+2 n+1) \beta_{2 j, n}+2 \beta_{2 j, n-1} \\
\beta_{2 j, n}=-(n+1) \beta_{2 j-1, n+1}+\beta_{2 j-1, n}
\end{array}\right.
$$

where $n=0,1,2, \ldots, j-1$.

Now we can compute the explicit formula of the general C-K extension

$$
C K(g)(\underline{x})=\sum_{\ell=0}^{\infty} \frac{\left(x_{1} \underline{e}_{1} \partial_{\underline{v}}\right)^{\ell}}{\ell !}\left(\underline{e}_{1} a(u) M_{k}(\underline{v})\right)+\sum_{\ell=0}^{\infty} \frac{\left(x_{1} \underline{e}_{1} \partial_{\underline{v}}\right)^{\ell}}{\ell !}\left(\underline{v} b(u) M_{k}(\underline{v})\right) .
$$

Denote the right-hand side of the above formula by

$$
I_{1}=\sum_{\ell=0}^{\infty} \frac{\left(x_{1} \underline{e}_{1} \partial_{\underline{v}}\right)^{\ell}}{\ell !}\left(\underline{e}_{1} a(u) M_{k}(\underline{v})\right)
$$

and

$$
I_{2}=\sum_{\ell=0}^{\infty} \frac{\left(x_{1} \underline{e}_{1} \partial_{\underline{v}}\right)^{\ell}}{\ell !}\left(\underline{v} b(u) M_{k}(\underline{v})\right) .
$$

Firstly, we compute $I_{1}$. Making use of Lemma 4 gives

$$
\begin{aligned}
I_{1}= & \sum_{j=0}^{\infty} \frac{x_{1}^{2 j+1}}{(2 j+1) !}\left(\underline{e}_{1} \partial_{\underline{v}}\right)^{2 j+1}\left(\underline{e}_{1} a(u) M_{k}(\underline{v})\right)+\sum_{j=0}^{\infty} \frac{x_{1}^{2 j}}{(2 j) !}\left(\underline{e}_{1} \partial_{\underline{v}}\right)^{2 j}\left(\underline{e}_{1} a(u) M_{k}(\underline{v})\right) . \\
= & \sum_{j=0}^{\infty} \frac{x_{1}^{2 j+1}}{(2 j+1) !}\left(2^{2 j+1} \sum_{n=0}^{j} \frac{(-1)^{j+n}\left(\begin{array}{c}
j \\
j-n
\end{array}\right) \Gamma\left(j+\frac{s+1}{2}\right)}{\Gamma\left(n+\frac{s+1}{2}\right)} \underline{v}^{2 n+1} a^{(n+j+1)}(u) M_{k}(\underline{v})\right) \\
& +\sum_{j=0}^{\infty} \frac{x_{1}^{2 j}}{(2 j) !}\left(2^{2 j} \underline{e}_{1} \sum_{n=0}^{j} \frac{(-1)^{j+n}\left(\begin{array}{c}
j \\
j-n
\end{array}\right) \Gamma\left(j+\frac{s-1}{2}\right)}{\Gamma\left(n+\frac{s-1}{2}\right)} \underline{v}^{2 n} a^{(n+j)}(u) M_{k}(\underline{v})\right) .
\end{aligned}
$$

Rearranging the summation above yields

$$
\begin{aligned}
I_{1}= & \sum_{n=0}^{\infty} \frac{(-1)^{n} 2^{2 n+1}}{\Gamma\left(n+\frac{s}{2}+\frac{1}{2}\right)} \underline{v} u^{n} x_{1}^{2 n+1} a^{(2 n+1)}(u) \sum_{j=0}^{\infty} \frac{(-1)^{j} 2^{2 j}\left(\begin{array}{c}
j+n \\
j
\end{array}\right) \Gamma\left(j+n+\frac{s+1}{2}\right)}{\Gamma(2 j+2 n+2)} x_{1}^{2 j} a^{(j)}(u) M_{k}(\underline{v}) \\
& +\sum_{n=0}^{\infty} \frac{(-1)^{n} 2^{2 n}}{\Gamma\left(n+\frac{s}{2}-\frac{1}{2}\right)} \underline{e}_{1} u^{n} x_{1}^{2 n} a^{(2 n)}(u) \sum_{j=0}^{\infty} \frac{(-1)^{j} 2^{2 j}\left(\begin{array}{c}
j+n \\
j
\end{array}\right) \Gamma\left(j+n+\frac{s-1}{2}\right)}{\Gamma(2 j+2 n+1)} x_{1}^{2 j} a^{(j)}(u) M_{k}(\underline{v}) .
\end{aligned}
$$

Denote

$$
\Phi_{\alpha, \beta, \kappa}\left(x_{1}, \partial_{u}\right)[f(u)]=\frac{2^{\kappa} x_{1}^{\kappa} \partial_{u}^{\kappa}}{(-1)^{\alpha} \kappa !}{ }_{1} F_{1}\left(\alpha+\frac{s+1}{2} ; \beta+\frac{1}{2} ;-x_{1}^{2} \partial_{u}\right) f(u)
$$

where ${ }_{1} F_{1}(a ; b ; z)=\sum_{k=0}^{\infty} \frac{(a)_{k}}{(b)_{k}} \frac{z^{k}}{k !}$ is the hypergeometric function. Then $I_{1}$ can be written as

$$
I_{1}=\sum_{n=0}^{\infty} \underline{v} u^{n} \Phi_{n, n+1,2 n+1}\left(x_{1}, \partial_{u}\right)[a(u)] M_{k}(\underline{v})-\sum_{n=0}^{\infty} \underline{e}_{1} u^{n} \Phi_{n-1, n, 2 n}\left(x_{1}, \partial_{u}\right)[a(u)] M_{k}(\underline{v}) .
$$

Similarly, we can obtain $I_{2}$ in terms of $\Phi$, which gives us the following result. 
Theorem 5. Let $\underline{x}=x_{1} \underline{e}_{1}+\underline{v}$ where $\underline{v} \in \mathbb{R}_{m-1}, u=|\underline{v}|^{2}$. Any function $g(\underline{v})$ of the form (15) has an axially monogenic extension $f(\underline{x})$ of the form (16) in which the function $A\left(x_{1}, u\right)$ can be written as

$$
\begin{aligned}
A\left(x_{1}, u\right)= & -(s-1) x_{1} \Phi_{0,1,0}\left(x_{1}, \partial_{u}\right)[b(u)]-\sum_{n=0}^{\infty}\left(u^{n} \Phi_{n-1, n, 2 n}\left(x_{1}, \partial_{u}\right)[a(u)]\right. \\
& \left.+\frac{1}{n+1} u^{n+1} \Phi_{n, n+1,2 n+1}\left(x_{1}, \partial_{u}\right)[b(u)]+\frac{2 n+1}{2 n+2} u^{n+1} \Phi_{n, n, 2 n+1}\left(x_{1}, \partial_{u}\right)[b(u)]\right),
\end{aligned}
$$

and the function $B\left(x_{1}, u\right)$ can be written as

$$
B\left(x_{1}, u\right)=\sum_{n=0}^{\infty}\left(u^{n} \Phi_{n, n+1,2 n+1}\left(x_{1}, \partial_{u}\right)[a(u)]+u^{n} \Phi_{n, n, 2 n}\left(x_{1}, \partial_{u}\right)[b(u)]\right),
$$

where $s=m+2 k$.

Remark 8. It is easy to check that the $C$-K extension $f(\underline{x})$ we obtained in Theorem 5 is monogenic and satisfies the restriction condition.

Remark 9. In the case when $g(\underline{v})=\left(a\left(|\underline{v}|^{2}\right)+\underline{e}_{1} \underline{v} b\left(|\underline{v}|^{2}\right)\right) M_{k}(\underline{v})$, the $C-K$ extension $f(\underline{x})$ in Theorem 4 can be written as

$$
f(\underline{x})=\left(A\left(x_{1},|\underline{v}|^{2}\right)+B\left(x_{1},|\underline{v}|^{2}\right) \underline{e}_{1} \underline{v}\right) M_{k}(\underline{v}) .
$$

Theorem 6. Let $\underline{y}=y_{1} \underline{e}_{1}+\rho \underline{\eta}, \underline{\eta} \in \mathbb{S}^{m-2}, f\left(y_{1}, \rho \underline{\eta}\right)=C K\left((\rho \underline{\eta})^{s} M_{k}(\rho \underline{\eta})\right)$. Then the Bargmann-Radon transform for the $\bar{C}-K$ extension $f\left(y_{1}, \rho \underline{\eta}\right)$ can be written as

$$
R_{\underline{\tau}}[f](\underline{x})= \begin{cases}\sigma_{k, 2 n}\left(x_{1}+i\langle\underline{x}, \underline{s}\rangle\right)^{2 n+k} \frac{\tau}{\tau} \frac{\tau}{\dagger}^{\dagger} M_{k}(\underline{s}), & s=2 n, \\ \sigma_{k, 2 n+1}\left(x_{1}+i\langle\underline{x}, \underline{s}\rangle\right)^{2 n+k+1} \underline{\tau} M_{k}(\underline{s}), & s=2 n+1 .\end{cases}
$$

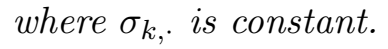

Proof. Recalling the Bargmann-Radon transform (5), we rewrite it as

$$
R_{\underline{\tau}}[f](\underline{x})=\frac{1}{(2 \pi)^{m / 2}} \sum_{\ell=0}^{\infty} \gamma_{\ell}\langle\underline{x}, \underline{\tau}\rangle^{\ell} \underline{\tau} \underline{\tau}^{\dagger} \int_{\mathbb{R}^{m}} e^{-|\underline{y}|^{2} / 2}\left\langle\underline{y}, \underline{\tau}^{\dagger}\right\rangle^{\ell} f(\underline{y}) d \underline{y} .
$$

We first consider the integral, denoted by $I$, in the above formula. Let $\underline{y}=r \underline{\omega}, \underline{\omega} \in \mathbb{S}^{m-1}$, then $|\underline{y}|^{2}=r^{2}$ and the integral can be written as

$$
I=\int_{0}^{+\infty} \int_{\mathbb{S}^{m-1}} e^{-r^{2} / 2} r\left\langle\underline{\omega}, \underline{\tau}^{\dagger}\right\rangle^{\ell} f(r \underline{\omega}) r^{m-1} d S(\underline{\omega}) d r .
$$

We recall that $f$ is a spherical monogenic function to the variable $\underline{y}$ of degree $k+s$. Then for fixed $r>0, f(r \underline{\omega})=r^{k+s} f(\underline{\omega})$ and

$$
I=\int_{0}^{+\infty} e^{-r^{2} / 2} r^{m+k+s} d r \int_{\mathbb{S}^{m-1}}\left\langle\underline{\omega}, \underline{\tau}^{\dagger}\right\rangle^{k+s} f(\underline{\omega}) d S(\underline{\omega}) .
$$

Due to the orthogonality of spherical monogenics, only the case of $\ell=k+s$ remains in the transform. Therefore, using cylindrical coordinates, we have

$$
\begin{aligned}
R_{\underline{\tau}}[f](\underline{x}) & =\frac{1}{(2 \pi)^{m / 2}} \gamma_{k+s}\langle\underline{x}, \underline{\tau}\rangle^{k+s} \underline{\tau} \underline{\tau}^{\dagger} \int_{\mathbb{R}^{m}} e^{-|\underline{y}|^{2} / 2}\left\langle\underline{y}, \underline{\tau}^{\dagger}\right\rangle^{k+s} f(\underline{y}) d \underline{y} \\
& =\frac{1}{(2 \pi)^{m / 2}} \gamma_{k+s}\langle\underline{x}, \underline{\tau}\rangle^{k+s} \underline{\tau} \underline{\tau}^{\dagger} \int_{\mathbb{R}} \int_{0}^{+\infty} \int_{\mathbb{S}^{m-2}} e^{-\frac{y_{1}^{2}+\rho^{2}}{2}}\left(-y_{1}+i \rho\langle\underline{\eta}, \underline{s}\rangle\right)^{k+s} f\left(y_{1}, \rho \underline{\eta}\right) d S(\underline{\eta}) d \rho d y_{1} .
\end{aligned}
$$


Similar to Theorem 2, we can obtain the Bargmann-Radon transform via the Funk-Hecke Theorem. Making use of the two forms (16) and (19) of the C-K extension $f\left(y_{1}, \rho \underline{\eta}\right)$, the transform can be written as

$$
R_{\underline{\tau}}[f](\underline{x})=\langle\underline{x}, \underline{\tau}\rangle^{k+s} \underline{\tau} \underline{\tau}^{\dagger}\left(T_{k, s} \underline{e}_{1}+S_{k, s} \underline{s}\right) M_{k}(\underline{s}),
$$

or

$$
R_{\underline{\tau}}[f](\underline{x})=\langle\underline{x}, \underline{\tau}\rangle^{k+s} \underline{\tau} \underline{\tau}^{\dagger}\left(T_{k, s}+S_{k, s} \underline{e}_{1} \underline{s}\right) M_{k}(\underline{s}),
$$

where $T_{k, s}$ and $S_{k, s}$ are constants. Furthermore, we have the relations

$$
\underline{\tau} \underline{\tau}^{\dagger} \underline{e}_{1} \underline{s}=-i \underline{\tau} \underline{\tau}^{\dagger}, \quad \underline{\tau} \underline{\tau}^{\dagger} \underline{e}_{1}=i \underline{\tau} \underline{\tau}^{\dagger} \underline{s}=2 \underline{\tau} .
$$

Making use of the relations above results in

$$
R_{\underline{\tau}}[f](\underline{x})= \begin{cases}\sigma_{k, 2 n}\left(x_{1}+i\langle\underline{x}, \underline{s}\rangle\right)^{2 n+k} \underline{\tau} \tau^{\dagger} M_{k}(\underline{s}), & s=2 n \\ \sigma_{k, 2 n+1}\left(x_{1}+i\langle\underline{x}, \underline{s}\rangle\right)^{2 n+k+1} \underline{\tau} M_{k}(\underline{s}), & s=2 n+1,\end{cases}
$$

which is the Bargmann-Radon transform for the C-K extension of the polynomial $(\rho \underline{\eta})^{s} M_{k}(\underline{\eta})$.

\section{Acknowledgment}

Ren Hu is supported by the China Scholarship Council [201708120045].

Alí Guzmán Adán is supported by a BOF-post-doctoral grant from Ghent University.

\section{References}

[1] G. E. Andrews, R. Askey and R. Roy, Special functions, Cambridge Univ. Press, Cambridge, Encyclopedia of mathematics and its applications, 2006

[2] H. Bateman, Higher transcendental functions. Volume 1, Krieger, Malabar, Fla, Bateman Manuscript Project, 1981

[3] V. Bargmann, On a Hilbert space of analytic functions and an associated integral transform part I, Communications on Pure and Applied Mathematics, 14, 1961, 187-214.

[4] F. Brackx, R. Delanghe and F. Sommen, Clifford analysis, Pitman Advanced Pub. Program, Boston, Research notes in mathematics, 1982

[5] J. Bures, R. Lavicka and V. Soucek, Elements of Quaternionic Analysis and Radon Transform, Textos de Matematica, 42, 2009, 52 .

[6] A. K. Common and F. Sommen, Axial Monogenic Functions from Holomorphic Functions, Journal of Mathematical Analysis and Applications, 179, 1993, 610-629.

[7] F. Colombo, I. Sabadini and F. Sommen, On the Szegö-Radon projection of monogenic functions, Advances in Applied Mathematics, 74, 2016, 1-22.

[8] F. Colombo, I. Sabadini and F. Sommen, On the Bargmann-Radon transform in the monogenic setting, Journal of Geometry and Physics, 120, 2017, 306-316.

[9] S. R. Deans, The Radon transform and some of its applications, Wiley, New York, 1983 
[10] R. Delanghe, F. Sommen and V. Souček, Clifford Algebra and Spinor-Valued Functions, Springer Netherlands, Dordrecht, 1992

[11] I. M. Gelfand and G. E. Shilov, Generalized functions, AMS Chelsea Publishing, American Mathematical Society, Providence, Rhode Island, 2016

[12] F. He, M. Ku, U. Kähler, F. Sommen and S. Bernstein, Riemann-Hilbert problems for monogenic functions in axially symmetric domains, Boundary Value Problems, 2016, 2016, 22

[13] H. Hochstadt, The Functions of Mathematical Physics, Wiley-Interscience, New York, 23, 1971

[14] S. Helgason, Integral geometry and Radon transforms, Springer, New York, 2011

[15] G. Jank and F. Sommen, Clifford analysis, biaxial symmetry and pseudoanalytic functions, Complex Variables, Theory and Application: An International Journal, 13, 1990, $195-212$.

[16] F. W. J. Olver and National Institute of Standards and Technology(U.S.), NIST handbook of mathematical functions, Cambridge University Press : NIST, Cambridge; New York, 2010

[17] D. Peña Peña, I. Sabadini and F. Sommen, Segal-Bargmann-Fock modules of monogenic functions, Journal of Mathematical Physics, 58, 2017, 103507.

[18] J. Radon, Über die Bestimmung von Funktionen durch ihre Integralwertelängs gewisser Mannigfaltigkeiten, 69, 1917, 262-277.

[19] F. Sommen, Radon and X-ray transforms in clifford analysis, Complex Variables, Theory and Application: An International Journal, 11, 1989, 49-70.

[20] F. Sommen, An extension of the radon transform to clifford analysis, Complex Variables, Theory and Application: An International Journal, 8, 1987, 243-266.

[21] F. Sommen, Plane elliptic systems and monogenic functions in symmetric domains, Proceedings of the 12th Winter School on Abstract Analysis, 1984, 259-269.

[22] F. Sommen, Special functions in Clifford analysis and axial symmetry, Journal of Mathematical Analysis and Applications, 130, 1988, 110-133.

[23] F. Sommen, Clifford Analysis and Integral Geometry, Springer Netherlands, Dordrecht, Fundamental Theories of Physics, 1992, 293-311.

[24] L. A. Shepp, Computed tomography, American Mathematical Society, Providence, R.I, Proceedings of symposia in applied mathematics, 1983

[25] I. N. Vekua, Generalized analytic functions, Pergamon Press, London-Paris-Frankfurt; Addison-Wesley Publishing Co., Inc., Reading, Mass., 1962

Alí Guzmán Adán

Clifford Research Group, Department of Electronics and Information Systems, Ghent University, Krijgslaan 281, 9000 Gent, Belgium

E-mail: Ali.GuzmanAdan@UGent.be

Ren $\mathrm{Hu}$ 
Clifford Research Group, Department of Electronics and Information Systems, Ghent University, Krijgslaan 281, 9000 Gent, Belgium

E-mail: Ren.Hu@UGent.be

Tim Raeymaekers

Clifford Research Group, Department of Electronics and Information Systems, Ghent University, Krijgslaan 281, 9000 Gent, Belgium

E-mail: Tim.Raeymaekers@UGent.be

Franciscus Sommen

Clifford Research Group, Department of Electronics and Information Systems, Ghent University, Krijgslaan 281, 9000 Gent, Belgium

E-mail: Franciscus.Sommen@UGent.be 